\title{
High-Speed Analysis of Pt Based Alloys at High Spatial Resolution using EELS
}

\author{
Paolo Longo ${ }^{1}$, R.D. Twesten ${ }^{1}$, M. Bugnet ${ }^{2}$, S. Prabhudev ${ }^{2}$ and G.A. Botton ${ }^{2}$ \\ ${ }^{1}$ Gatan Inc. R\&D, 5794 W Las Positas Blvd., Pleasanton CA, 94588, USA \\ ${ }^{2}$ Department of Materials Science \& Engineering, McMaster University, Ontario, Canada L8S \\ 4M1
}

Platinum based nano-particles with a narrow size distribution are very important from a technological point of view due to their chemical and physical properties and applications in the field of catalysis, information storage, membrane exchange fuel cells etc. However, their composition and size distribution influence their chemical and electrical properties and also the surface activity. This is the reason why a lot of attention has been paid on the development of synthesis methods capable to control the size, the composition and elemental distribution across the nanoparticles. Hence, the study of the chemistry and the elemental distribution is important in order to understand the properties of the nano-particles systems. Analytical transmission electron microscopy (TEM) related techniques have proved to be valuable tools for characterizing such materials.

Data was acquired using a probe and image corrected FEI Titan STEM operating at $300 \mathrm{kV}$ (McMaster University). The microscope is also equipped with a fully upgraded GIF Quantum ERS as EELS spectrometer. Two different sets of samples were analyzed. The first one is Pt-Fe catalyst nanoparticles and the second one is $\mathrm{TiO}_{\mathrm{x}}$ supported Pt-Ru core-shell catalyst nanoparticles. Both sets of samples were deposited on a carbon film supported on a Cu mesh TEM grid. EELS data was acquired in DualEELS mode in order to have both the low- and core-loss regions in the case of the Pt-Fe nanoparticles and to have two different core-loss regions at high energy for the collection of the Pt $\mathrm{M}_{4,5}$-edges at $2120 \mathrm{eV}$ and the $\mathrm{Ru} \mathrm{L}_{2,3}$-edges at $2838 \mathrm{eV}$ and at low energy for the acquisition of the $\mathrm{Ti} \mathrm{L}_{2,3}$-edges at $456 \mathrm{eV}$ and the $\mathrm{Ru} \mathrm{M}_{4,5}$-edges at $279 \mathrm{eV}$. In the case of the Pt-Ru catalyst system, provided there is enough signal, it is clearly more advantageous the collection of the $\mathrm{Ru} \mathrm{L}_{2,3}$-edges at $2838 \mathrm{eV}$ given the high peak-to-background ratio and also the fact that it does not overlap with other edges.

Figure 1 shows the color-coded maps obtained from a large Pt-Fe catalyst particle with a diameter of about $60 \mathrm{~nm}$ selected because of the large field of view and fine sampling were required to study the surface chemistry and distribution of elements simultaneously. EELS spectra were acquired using an exposure time of $5 \mathrm{~ms}$, a step size of about $0.1 \mathrm{~nm}$ and the entire area was mapped within a few minutes. It is interesting to notice the presence of a nicely defined $\mathrm{FeO}_{\mathrm{x}}$ as a shell layer. In Figures 2a,b, EELS spectra extracted from the outer shell, inner shell and core regions in the particle show that the shell consists of $\mathrm{FeO}_{\mathrm{x}}$. The compositional analysis obtained from EELS quantification across the entire particle shows Pt at $40 \%$ and $\mathrm{Fe}$ at $60 \%$. The projected concentration of Fe seems to increase slightly when measurements are made towards the shell region. The same type of analysis was carried out across other particles of much smaller dimensions with a diameter of approximately $10 \mathrm{~nm}$. Here the $\mathrm{FeO}_{\mathrm{x}}$ shell layer seems to be thinner and less pronounced than in the case of the $60 \mathrm{~nm}$ particle. The composition was measured using EELS and appears to be Pt at $60 \%$ and $\mathrm{Fe}$ at $40 \%$. The composition seems to be size dependent. Fe seems to be more abundant in large particles and as result the $\mathrm{FeO}_{\mathrm{x}}$ shell layer becomes more pronounced

Advances in electron sources, spectroscopic detectors and software control now make it possible to acquire EELS data from an analytical STEM with high efficiency for heavy elements and 
high-energy edges. Moreover, the acquisition of the data using the DualEELS capability allows the dynamic range to be increased and the possibility to correct for thickness and diffraction effects that can be significant for heavy materials.

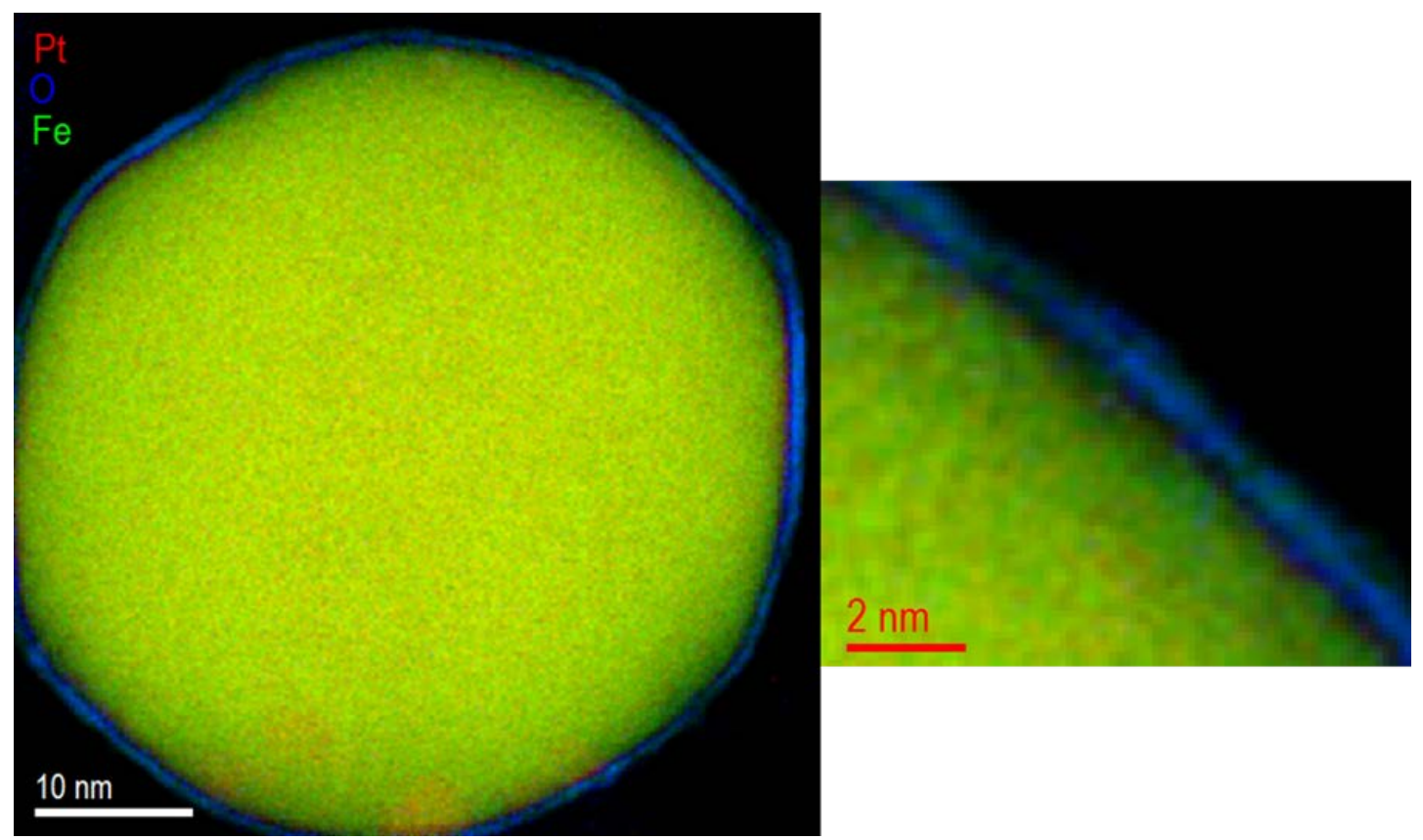

Figure 1: RGB composite image of $\mathrm{Pt}_{4,5}$-edges at $2120 \mathrm{eV}$ in red, $\mathrm{O}$ K-edge at $532 \mathrm{eV}$ in blue and $\mathrm{Fe}$ $\mathrm{L}_{2,3}$-edges at $708 \mathrm{eV}$ in green. The shell layer appears to be very well pronounced and show the presence of $\mathrm{Fe}$ and $\mathrm{O}$ only. The inset shows the region at the interface with the shell layer in more details.
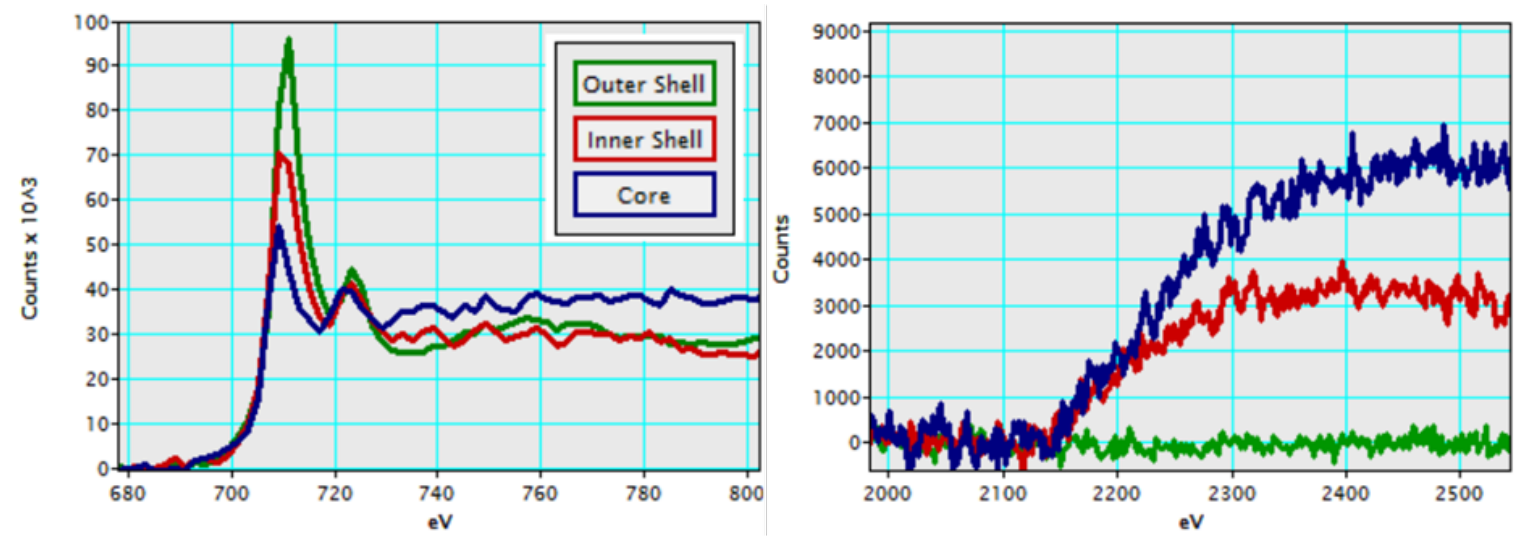

Figures 2: a) $\mathrm{Fe} \mathrm{L}_{2,3}$-edges at $708 \mathrm{eV}$ and b) $\mathrm{Pt}_{4,5}$-edges at $2120 \mathrm{eV}$ extracted from the core, inner and outer shell respectively. It is quite remarkable to see how the shape of the $F e \mathrm{~L}_{2,3}$-edges changes across the three regions. This could be an indication that the Fe is chemically changing across the particle 